\title{
P 011 PALLIATIVE AND END OF LIFE CARE IN PRISONS IN ENGLAND AND WALES - APPROACHES TAKEN TO IMPROVE INEQUALITIES
}

Andrew Fletcher, ${ }^{1}$ Sheila Payne, ${ }^{2}$ David Waterman, ${ }^{3}$ Mary Turner ${ }^{2} .{ }^{1}$ St Catherine's Hospice, Preston, UK; ${ }^{2}$ Lancaster University, Lancaster, UK; ${ }^{3}$ Stockport NHS Foundation Trust, Stockport, UK

\subsection{6/bmjspcare-2014-000654.52}

Background The End of Life Care Strategy (2008) states that healthcare providers, prisons and coronial services should develop strategies that enhance delivery of palliative and end of life care. The prison environment and rules potentially creates inequalities in care. A paucity of research means that although palliative care is recognised as desirable, there is a lack of evidence about the nature of what is required and its feasibility in a prison context.

Aims and Methods The aim of the study was to quantify palliative and end of life care need in adult prisons in England and Wales and identify services delivered and strategies to enhance care. A self-reported questionnaire was developed and consent for participation sought from 124 prisons.

Results Data obtained from 39 prisons (31.5\% response rate) indicate that between 2010-2012, 113 prisoners died from natural causes; retrospective review concluded that 68 required palliative care input. Forty-five were considered for release to receive care in an alternative location; 20 of them were released. Factors preventing release included on-going risk, failure to identify patients in a timely manner or obtain the required medical report, and prisoners' wishes to remain in prison. Two-thirds of prisons had a palliative care policy; 28\% had implemented the Gold Standards Framework and 36\% Advance Care Planning. Ninety percent of prisons had access to palliative care nurse specialists and $77 \%$ palliative medicine consultants. Three broad approaches were identified; the individual prison approach, the cluster approach and the regional approach.

Conclusions There is evidence of support from specialist palliative care services, implementation of palliative care principles and access to education for prison staff. The study has highlighted the challenges in developing palliative care services across the entire prisons service and the unique approaches adopted. Further research is underway to develop a model of integrated palliative and end of life care for dying prisoners. 